\title{
Brüchige Widerständigkeit. Zu Entwicklung und Grenzen des Konzepts der „immateriellen Arbeit“ in Hardt/Negris Multitude
}

MARC ZIEGLER

Dieser Aufsatz behandelt das Konzept der immateriellen und affektiven Arbeit, wie es vor allem in den Texten von Michael Hardt und Antonio Negri entfaltet wird. Es wird diskutiert, ob und inwiefern der theoriestrategische Fokus auf das Konzept der immateriellen Arbeit das zeitgenössische Denken der politischen Widerständigkeit zu strukturieren vermag. ${ }^{1}$ Zunächst betrachte ich dieses Konzept in seinen politischen, historischen und theoriegenetischen Kontexten (1). Von da aus wird ersichtlich, dass der Begriff der immateriellen Arbeit ein zentrales Konzept des widerständigen Denkens von Negri und Hardt darstellt (2). Abschließend wird der Ansatz von Negri/Hardt kritisch beleuchtet. Meine These ist, dass die immaterielle Arbeit sich nicht ungebrochen als ein gesellschaftliches Verhältnis der politischen Widerständigkeit verstehen lässt. $\mathrm{Zu}$ sehr zeigt sie sich in die Dynamik kapitalistischer Verwertungslogik eingebunden, zu wenig wird dabei von Negri/Hardt auf den immanenten Umschlag von kritischem Impuls in systemstabilisierende Konformität reflektiert. Geschuldet ist die interne Brüchigkeit des Widerstandskonzepts einem fehlenden normativen Rahmen, der die von Hardt und Negri betriebene sozial-ontologische Aufwertung der Multitude rechtfertigen könnte (3).

1 Zur allgemeinen Diskussion um das Konzept der immateriellen Arbeit vgl. Atzert/Müller (2004). 


\section{Kontexte}

Der Ausdruck Operaismus bezieht sich auf eine Gruppe Intellektueller und Arbeiter, die in den 60er und 70er Jahren des 20. Jh. vor allem im nördlichen Italien politisch aktiv geworden sind. Das Wort Operaismus lässt sich nur ungeschickt ins Deutsche übersetzen, wo es soviel heißt wie ,Arbeiterismus‘. Die Gruppenbezeichnung entwickelte sich im Zusammenhang mit den Massendemonstrationen der italienischen Industriearbeiter jener Zeit, die sich mit zunehmendem Selbstbewusstsein als eine Potere Operario, als eine Arbeitermacht, verstanden haben. Der Begriff Operaismus verweist damit auf ein Selbstverständnis politischer Widerständigkeit, und zwar unabhängig davon, ob sich dies in Form der Beteilung an einem Streik oder in Form politischer Reflexion artikuliert findet.

Das gemeinsame Zentrum der operaistischen Handlungen galt dem Versuch, Widerstand gegenüber kapitalistischen Formen der Repression zu leisten. Die Operaisten vor allem der 60er Jahre sahen in der fordistischen Arbeitsorganisation der großen Industrie ${ }^{2}$ den hauptsächlichen Zugriffspunkt kapitalistischer Ausbeutung und Kontrolle. Erklärtes Ziel war die Abschaffung der Lohnarbeit und damit einhergehend die Abschaffung kapitalistischer Kontrolle. Das knechtische Leben in der Fabrik sollte einem Leben in Freiheit, d.h. in politischer, sozialer und ökonomischer Selbstbestimmung weichen. Die entsprechenden Widerstandsformen suchten und fanden die Arbeiter und Intellektuellen in den verschiedenen Formen der Arbeitsverweigerung (Streik, Dienst nach Vorschrift, Nichterscheinen am Arbeitsplatz, das sich Verschaffen zusätzlicher Pausen). 1969 erreichte die Arbeiterbewegung einen vorläufigen Höhepunkt, als die Arbeiter der Turiner Fiat-Werke autonom agierende Arbeiterräte zu gründen begannen. Der italienische Staat reagierte mit einer „Strategie der Spannung“, die darin bestand, die Sicherheit zu erhöhen und mit Gewalt gegen die Potere Operario vorzugehen. Dies führte auf der Gegenseite ebenfalls zu einer Bewaffnung der Bewegung, was durch die 70er Jahre hindurch bürgerkriegsähnliche Zustände in Italien auslöste. Begleitet wurde dies mit dem Aufkommen der Autonomia, der italienischen Autonomiebewegung, in die schließlich auch der Operaismus aufging. Galt bislang nur die Fabrik als der umkämpfte Raum des sozialen Antagonismus, so entgrenzte sich der Kampf zusehends, trat über die Fabrikmauern hinaus und dehnte sich zusehends auf die ganze Stadt aus.

Die Wirtschaft Italiens reagierte auf die andauernde Krise mit einer zunehmenden Automatisierung der Fabriken, was es erlaubte, einen Großteil der widerständigen Arbeiterinnen und Arbeiter zu entlassen. Das wiederum löste eine Massenarbeitslosigkeit aus und war mitunter ein Faktor, der mit zur Wirtschaftskrise des Landes beitrug. Eine weitere Folge war die Zuspitzung des Konflikts

2 Als Kriterien fordistischer Arbeitsorganisation sind hier vor allem zu nennen: Standardisierte, mechanistische Arbeitsabläufe, Fließbandproduktion, fehlende Mitbestimmungsrechte der Arbeiter in der Produktion, dafür streng hierarchisch gegliederte und ,von oben' kontrollierte Arbeitsabläufe sowie fehlende Gruppenarbeit. 
zwischen den Gewerkschaften und dem mehrheitlich radikaleren Teil des Widerstandes. Während die Gewerkschaften zur Lösung des Konflikts eine Ausdehnung der Arbeitswoche auf den Samstag vorschlugen, blieben die widerständigen Arbeiter bei ihrem Ruf nach der Abschaffung der Lohnarbeit. Die allgemeine Instabilität umfasste also gleichermaßen Politik und Wirtschaft. 1977 kam es daher zu einem Schulterschluss zwischen der Christdemokratischen Partei und den Kommunisten. Die Autonomiebewegung geriet dadurch in eine Sackgasse, gleichzeitig entwickelte sich mit den Brigate Rosse, den Roten Brigaden, eine terroristische Gruppierung, deren Aktivitäten zunächst stark von den Autonomen begrüßt wurde.

Dies änderte sich, als 1979 der Vorsitzende der Christdemokraten, Aldo Moro von den Roten Brigaden nicht nur entführt, sondern auch getötet wurde. Tötungen gehörten bis dahin nicht zum Programm der Roten Brigaden. Dieser terroristische Akt kann als definiter Wendepunkt in der Entwicklungsgeschichte des italienischen Widerstands angesehen werden. Einige Monate nachdem im Mai 79 Moros Leiche gefunden wurde, antwortete der Staat mit einer breit angelegten Ermittlungs- und Verhaftungswelle. Es wurde gegen 60.000 Menschen ermittelt, 25.000 davon wurden inhaftiert. Dank schnell eingerichteter Sondergesetze konnte die Untersuchungshaft über Jahre andauern. ${ }^{3}$

Damit wären die für ein historisches Verständnis des Operaismus grundlegenden Eckdaten genannt. Der Operaismus im engeren Sinn lässt sich auf eine Gruppe engagierter Intellektueller beziehen, die sich seit 1961 über die Zeitschrift Quaderni Rossi, den „Roten Heften“, als eine publizierende Intellektuellengruppe artikulieren. Raniero Panzieri und Mario Tronti können als die Gründer der „Roten Hefte“ betrachtet werden. Die 60er Jahre dienten den Operaisten zur Entfaltung ihrer theoretischen Grundlagen, die sie im engen Umgang mit der Arbeiterschaft entwickelt haben und im wesentlichen in einer industriesoziologisch mitmotivierten Neuauslegung von Marx' Kapital bestand. Das Verhältnis von Arbeitskraft, Wertbildung und kapitalistischer Produktionsweise sollte nicht von dem kapitalistischen Akkumulationsbestreben aus, sondern von der produktiven Kraft des Widerstands her erklärt werden. Die Operaisten sahen in den sozial herbeigeführten Störungen den Motor für ökonomische, technologische und soziale Entwicklungen. Den sozialistischen Vorstellungen einer Planwirtschaft setzten sie die Forderung nach dem autonom agierenden Arbeiter, der in Kooperation mit anderen autonomen Arbeitern zur neuen Quelle gesellschaftlicher Produktivität wird, entgegen. Gleichfalls sollte nicht der kapitalistische Herrschaftsapparat den Arbeiter kontrollieren, sondern die aus der Zusammenarbeit der Arbeiter entstehenden Bedürfnisse sollten von sich aus zu einer sozialen Bändigung des an sich wilden Marktes führen (vgl. Ziegler 2004: 302-305).

3 Diese Ausführungen basieren auf der Filmdokumentation Antonio Negri-Eine Revolution, die nicht endet (Weltz/Pichle 2004). 
Was den Operaismus über ein sozialhistorisches Interesse hinaus heute aktuell erscheinen lässt, ist der Umstand, dass die Operaisten sozusagen politische Zeugen des wirtschaftlichen Wandels vom Fordismus zum Postfordismus in den 70er Jahren an den Stätten der Produktion selbst wurden. Die Nähe zu der Arbeiterschaft, die durch den gemeinschaftlichen politischen Kampf vorhanden war, konnten die Operaisten für die Fortführung des Kampfes mit theoretischen Mitteln im Rahmen einer Industriesoziologie nutzen, die sich wesentlich von der damals gängigen unterschied. Sie hatten auf mikrologischer Ebene Einblicksmöglichkeiten in die Gestaltung des Arbeitstags, sie konnten bis in die letzten düsteren Winkel der Fabrik schauen und somit verstehen lernen, mit welchen auffälligen oder unauffälligen Methoden die Arbeiter sich dem kapitalistischen Kommando fügen bzw. zu entziehen verstanden. Sie befanden sich in den , heißen' Zonen des Antagonismus von Kapital und Arbeit. Man könnte aus der Perspektive heutiger empirischer Sozialforschung sagen, dass die operaistische Doppelrolle, Arbeitskämpfer und politischer Theoretiker in Personalunion zu sein, eine Form umfassender teilnehmender Beobachtung ermöglichte, die den üblichen Rahmen der Arbeits- und Industriesoziologie bei weitem überstieg. Wohlverstanden ging es den Operaisten nicht in erster Linie darum, den akademischen Diskurs voranzutreiben: Das intellektuelle Interesse stand stets unter den Vorzeichen politischer Intervention. ${ }^{4}$

\section{Die vereinigende Kraft der immateriellen Arbeit}

Der Übergang von fordistischen zu postfordistischen Formen der Arbeitsorganisation vollzog sich in der Industrie Norditaliens in den 70er Jahren. Aus Sicht der Operaisten lässt sich dieser Übergang als eine Folge des Arbeitskampfs der vorangegangenen Jahre verstehen. Wie schon erwähnt, reagierte die große Industrie auf den Widerstand mit einer zunehmenden Automatisierung der Arbeitsabläufe und mit Massenentlassungen ehemals widerständiger Arbeiter. So entließ zu Beginn der 70er Jahre die Chemieindustrie Norditaliens 10.000 Arbeiter, was ungefähr einem Drittel der über die fünfziger und sechziger Jahre dort angestellten Arbeitskräfte entsprach (vgl. Negri 1998: 24). Antonio Negri hat untersucht, inwiefern die in die Arbeitslosigkeit getriebene Arbeiterschaft sich politisch weiterhin organisiert hat, wie sich dadurch aber auch neue Formen kleinerer und mittlerer Industrie herausbilden. Mit dem Aufkommen dieser neuen Betriebe verband sich auch das Auftreten eines neuen Typs von Arbeiter; dessen flexible

4 So schreibt Antonio Negri: „Mein Hintergrund ist nicht der eines berufsmäßigen Soziologen; ich spreche vielmehr vor allem als ein politischer Militanter aus den siebziger Jahren in Italien. Ich denke, dass diese Erfahrung deshalb einige Bedeutung hat, weil sie sich inmitten tiefgreifender Veränderungen der Produktionsweise entwickelte. Diese Veränderungen wiederum berühren die Praxis, die theoretische Reflexion wie die Möglichkeiten der politischen Intervention“" (Negri 1998: 23). 
Arbeitszeit nicht mehr den strikten Reglements der großindustriellen Firma entsprach; dessen Arbeit dadurch mobil wurde, dass er mit anderen Produzenten der kleinen und mittleren Industrie Kontakte aufnehmen und pflegen musste; und dessen Kompetenzbereich sich nicht mehr in dem wiederholten Vollzug standardisierter mechanischer Handlungen erschöpfte, sondern sich auf sprachliche und kommunikative Fähigkeiten ausdehnte. Mit anderen Worten: Durch die Automatisierung der Großbetriebe und die Schaffung dezentrierter Produktionsformen versuchte einerseits das großunternehmerische Kapital weitere, den Produktionsprozess störende politische Unruhen zu vermeiden; andererseits führte dies zur Bildung neuer, dezentraler Formen der Arbeitsorganisation und neuer Arbeitstypen, die Negri zunächst unter den Begriff des „gesellschaftlichen Arbeiters“, dann aber auch als ,politischen Unternehmer“ (Negri 1998: 31) fasst. Nach Negri arbeitet der politische Unternehmer, ,in einem Ensemble institutioneller Faktoren, die einer industriellen Tätigkeit vorausgesetzt sind, darin die Reproduktion ermöglichen, die Ausbildung der Arbeitskraft, die gesellschaftliche Kooperation. Der politische Unternehmer agiert autonom in einer immer stärker kooperativen, auf die territoriale Produktion bezogenen gesellschaftlichen Arbeit" (Negri 1998: 31). Dabei eröffnet sich dem neuen Arbeitstyp ein erweiterter Handlungsspielraum, der von Negri als ein Zuwachs von Autonomie und Unabhängigkeit verstanden wird:

Einheiten, die verteilt im Territorium arbeiten, die Produktionskosten auf jede nur erdenkliche Art senken und dabei Verwandte und Kinder bisweilen erbarmungslos ausbeuten, schlagen jede Konkurrenz. Aus jedem Parterre - die Häuser der Arbeiter sind aus Ersparnissen gebaut, die aus der Emigration oder aus den ,guten Zeiten' in festen Arbeitsverhältnissen stammen - wird eine Werkstatt. Aber die Konkurrenzfähigkeit erwächst nicht aus dem Drücken der Produktionskosten. Die Erfahrung der Leute bezieht sich nicht nur auf die Arbeitsorganisation, sie haben auch keine Probleme, sich in das Auto oder den Zug zu setzen, loszuziehen und neue Märkte zu suchen, sich von der Protektion der alten Patrone loszumachen, die Produktion zu regulieren, etc. Im gleichen $\mathrm{Ma}$ werden die neuen kleinen und mittleren Betriebe, die aus der Dezentralisierung hervorgingen, unabhängig und selbständig, alles in kürzester Zeit. Mit der zunehmenden Unabhängigkeit gehen diese politischen Unternehmer Formen der Kooperation mit anderen Unternehmern ein, assoziieren sich und bedienen sich aller anderen Instrumente, um ihre Tätigkeit auszudehnen und zu konsolidieren. (Negri 1998: 32f.)

Negri konstatiert hier eine Verschiebung in der gesellschaftlichen Arbeitsproduktion, anhand derer neue Produktivquellen der Arbeitskraft analysierbar werden, die bislang gerade den fordistischen Produktionszyklen weitestgehend unbekannt waren: Flexibilität, Mobilität, Initiativhandlungen, kommunikatives Geschick, Einbezug der Affekte in den Arbeitsprozess sowie kreatives und kooperatives Verhalten des Arbeiters. War die fordistische Phase der Warenproduktion durch manuelles Arbeiten im Rahmen der großen Industrie bestimmt, so zeichnet sich im Übergang zum Postfordismus eine Neubewertung des Umgangs mit Wis- 
sens als die hauptsächliche gesellschaftliche Produktivkraft ab. Negri und unter anderem Maurizio Lazzarato (vgl. Lazzarato 1998) entwickeln vor diesem Hintergrund den Begriff der immateriellen Arbeit, der in der Folgezeit zum Schlüsselbegriff für den Postoperaismus vor allem der 90er Jahre wird. Der Begriff der immateriellen Arbeit wird schließlich auch in Hardt/Negris Gegenwartsdiagnose Empire (Hardt/Negri 2003) zum zentralen Konzept, von dem aus das Autorenteam den Widerstand der Multitude denkt. In ihrem Buch Multitude (Hardt/Negri 2004) unterscheiden Hardt/Negri zwei Formen der immateriellen Arbeit: Zum einen eine intellektuelle und sprachliche Dimension, die mit der „Lösung von Problemen“ konfrontiert ist. „Diese Art immaterieller Arbeit bringt Ideen, Symbole, Codes, Texte, sprachliche Figuren, Bilder und Ähnliches hervor" (Hardt/ Negri 2004: 126). Zum anderen besteht immaterielle Arbeit für Negri und Hardt in ,affektiver Arbeit“. Dabei fassen sie den Begriff des Affektes weiter als den des Gefühls:

Im Gegensatz zu Gefühlen, die psychische Phänomene sind, betreffen Affekte Körper und Psyche gleichermaßen. Tatsächlich lassen Affekte wie Freude oder Traurigkeit den Zustand erkennen, indem sich der ganze Organismus gerade befindet, da sich in ihnen ein bestimmter körperlicher Zustand und eine bestimmte Art und Weise des Denkens ausdrücken. Affektive Arbeit ist somit die Arbeit, die Affekte wie Behagen, Befriedigung, Erregung oder Leidenschaft hervorbringt oder manipuliert. (Hardt/Negri 2004: 126)

Die Autoren legen es nahe, die immaterielle und affektive Arbeit als biopolitische Arbeit zu verstehen. Letzten Endes geht es Negri und Hardt darum, in diesen neuen Formen der Arbeit eine Bewegung auszumachen, die sie als das „Gemeinsam-Werden der Arbeit“ bezeichnen und die für sie zur Grundvoraussetzung der widerständigen Multitude wird.

Die These lautet [...], dass die vielen singulären Beispiele für Arbeitsprozesse, Produktionsbedingungen, lokale Situationen und lebendige Erfahrungen auf einem anderen Abstraktionsniveau als ,Gemeinsam-Werden' der Formen der Arbeit und der Produktions- und Distributionsverhältnisse im Allgemeinen charakterisiert werden können - es gibt keinen Widerspruch zwischen dieser Singularität und der Gemeinsamkeit. Das Gemeinsam-Werden, das die qualitativen Unterschiede innerhalb der Arbeit immer geringer werden lässt, ist die biopolitische Bedingung der Multitude. (Hardt/Negri 2004: 133)

Eine solche Konzeption des Arbeitsbegriffs erschließt den Widerstandsbegriff der Multitude. Hardt/Negri unternehmen den Versuch, die widerständige Vielheit als eine in sich plurale, nicht zu vereinheitlichende Klasse zu denken, die sich aus einer Reihe von Singularitäten zusammensetzt (vgl. Hardt/Negri 2004: 117) und die sich auf das, was die Autoren das „Gemeinsam-Werden“ nennen, hin produziert. Hardt/Negri spielen dabei mit einer Fleisch- und Körpermetaphorik, wobei 
das lebendige, nicht kontrollierbare biopolitisch erzeugte und sozusagen soziale Fleisch der Multitude dem souveränen Kontrollkörper des imperialen Kapitalismus gegenüber gestellt wird: „Aus sozioökonomischer Sicht ist die Multitude das allgemeine Subjekt der Arbeit, also das wahre Fleisch der postmodernen Produktion, und zur gleichen Zeit ist sie das Objekt, aus dem das Gesamtkapital den Körper seiner globalen Entwicklung zu machen sucht“ (Hardt/Negri 2004: 119). Während der kapitalistische Körper durch Hierarchiebildung herrscht, soll das lebendige Fleisch der Multitude in der freien Artikulation der Differenzen ohne jegliche interne Hierarchiebildung sich entfalten. Unterschiede wie Rasse, Geschlecht und Sexualität sollen dann zwar weiter bestehen, doch nicht mehr in ihrer Form als sozial vermittelte Machthierarchien (Hardt/Negri 2004: 119).

\section{Neue Ausbeutungen im Namen der immateriellen Arbeit}

Bemerkenswert erscheint hierbei allerdings der Umstand, dass Negri schon in den 90er Jahren zwar die erweiterten Handlungsspielräume des ,,politischen Unternehmers" lobend hervorhebt, aber die mit ihm einhergehenden neuen Ausbeutungsverhältnisse nur am Rande berührt. Negri unterliegt in seinen Folgeveröffentlichungen zunehmend der Tendenz, die Zunahme an Autonomie und Unabhängigkeit der Widerständigkeit der Arbeitskraft zuzusprechen, wohingegen die gleichzeitig mitentstehenden neuen Formen von Gewalt, Repression, Kontrolle, Einschränkung der Handlungsspielräume und Ausbeutung dem Wirken des kapitalistischen Regimes zugewiesen werden. Negri und Hardt unternehmen sowohl in Empire als auch in ihrem jüngsten Werk Multitude in immer neuen Anläufen Polarisationsversuche, die Unterscheidung zwischen einer als positiv erachteten vielfältigen produktiven Widerständigkeit und einer als negativ bewerteten ebenso vielfältigen Ausbeutung und Unterdrückung in der jeweils gleichen Gestalt der Arbeit zu stabilisieren. Sowohl in ihrer Machtanalyse als auch in der sich daran anschließenden Ontologie des sozialen Lebens führen Negri/Hardt diese Polarisation aus: Die kriegsführende Biomacht steht da der auf Kommunikation und Kooperation beruhenden biopolitischen Produktion der lebendigen Arbeit gegenüber, und dem Unsicherheit und Angst kommunizierenden ontologisch leeren Empire opponiert die mit Affekten und Begehren ontologisch aufgeladene Produktivität der Multitude. So einleuchtend auch diese Zuweisung zunächst erscheinen mag, so fragwürdig bleibt dabei, inwiefern nicht die Kooperation, Kommunikation und Affekte der Multitude durch die kapitalistische Produktionsweise mitgeformt und durch sie vermittelt bleiben. Indem Negri und Hardt scharfsinnig die Verschiebung der Herrschaftsmechanismen im Hinblick auf Arbeitsverhältnisse aufzeichnen, leugnen sie geradezu die eigensinnige Produktivität des Kapitalismus. Eigentümlich genug: Sie hintergehen den Umstand, dass es nicht nur die Widerständigkeit der Ausgebeuteten ist, die global zu einer 
Transformation der Arbeits- und Lebenswelten führt, sondern dass es zur Eigenlogik des Kapitalismus gehört, sich strategisch erfolgreich auf veränderte Formen der Arbeitsorganisationen zu beziehen und diese für sich arbeiten zu lassen. Waren es in den 60er und 70er Jahren die am fordistischen Modell der Arbeitsorganisation ausgerichteten Forderungen nach mehr Eigenverantwortlichkeit, Kreativität und Selbstbestimmung, die sich die Operaisten auf die revolutionären Fahnen geschrieben haben, so lässt sich aus heutiger arbeitssoziologischer Perspektive nachweisen, dass die damals ins Feld geführten Kritikpunkte vom postfordistischen Kapitalismus in spezifischen Formen inkorporiert wurde. Was damals als Kritik formuliert wurde, findet sich heute - folgt man beispielsweise den Analysen des französischen Soziologenteams Luc Boltanski und Ève Chiapello als Selbstlegitimation des Kapitalismus wieder (vgl. Boltanski/Chiapello 2003).

Paolo Virno, ein Mitstreiter Negris, hat aus diesem Umstand 1998 den Schluss gezogen, dass die Antwort des Kapitalismus auf die revolutionären Forderungen der potere operario der 60er Jahre und der Autonomiebewegung in den 70er Jahren die Gestalt einer Konterrevolution angenommen hat (vgl. Virno 1998):

Die Konterrevolution geht von den gleichen Voraussetzungen, den gleichen (ökonomischen, sozialen oder kulturellen) Tendenzen aus, auf die sich auch eine Revolution stützen würde: Sie besetzt und kolonisiert das Terrain ihrer Gegnerin, indem sie entgegengesetzte Antworten auf die gleichen Fragen gibt. In ihr wird, in anderen Worten, das ganze Set materieller Bedingungen, die etwa die Abschaffung der Lohnarbeit vorstellbar machten, reinterpretiert, so dass all diese Tendenzen und Produktivkräfte profitbringend eingespannt werden konnten. [...] Darüber hinaus kehrt die Konterrevolution eine Praxis der Massen um, die die Staatsmacht schwächte und die darin die Möglichkeit radikaler Selbstverwaltung zu enthalten schien, und transformiert sie in eine entpolitisierte Passivität beziehungsweise einen plebiszitären Konsens. (Virno 1998: 83f.)

Die Folgerung, die Virno daraus zieht, lässt allerdings den Brückenschlag zu Negri und Hardt zu: „Eine kritische Geschichtsschreibung, die sich weigert, die sogenannten Tatsachen anzubeten, ist deshalb gezwungen, in jedem Schritt und jedem Aspekt der Konterrevolution die Umrisse, die Inhalte und die Bedingungen der revolutionären Möglichkeit zu entdecken“ (Virno 1998: 84).

Die Konterrevolution bezieht sich danach also auf die gleiche ökonomische Ausgangssituation wie eine mögliche Revolution, nur dreht sie in ihrer Interpretation die Vorzeichen um: Aus Emanzipation wird neuer Profit geschlagen. Aber aus diesem Double-Dasein der Konterrevolution sollen nun, in einem weiteren Schritt, Möglichkeiten neuer Widerstandsformen sichtbar werden.

Doch an diesem Punkt werden die ansonst sehr weitläufig und feinsinnig durchgeführten Analysen von Hardt und Negri in Empire und Multitude unscharf: Der Unterschied zwischen den Formen kapitalistischer Selbstlegitimation und Formen der Kapitalismuskritik droht zu implodieren. Denn gegen die politi- 
schen Absichten Negris und Hardts lässt sich der für das postoperaistische Projekt zentrale Begriff der immateriellen Arbeit nicht ausschließlich als eine Kategorie politischer, kultureller und ökonomischer Widerständigkeit fassen. Er dient heute gleichfalls der Selbstlegitimation kapitalistischer Herrschaftsstrukturen (vgl. Boltanski/Chiapello 2003) sowie der kapitalistischen Wertschöpfung (vgl. Möller 2005) und verknüpft die auf Kreativität, Innovation und Kommunikation ausgerichtete Subjektivität der Menschen ungleich stärker mit dem Arbeitsprozess, als dies in fordistischen Arbeitsverhältnissen noch der Fall war (vgl. Voß/Pongratz 1998; Moldaschl/Voß 2003 sowie Kels/Vormbusch 2005). Die intellektuellen Akteure des Postoperaismus weichen einer Auseinandersetzung mit diesen Umständen aus, in dem sie eine radikale Trennbarkeit von kapitalistischer Herrschaft des Empires und widerständigem Verhalten der Multitude postulieren und sozialontologisch unterfüttern (vgl. Ziegler 2004). Damit wird ein Denken der sozialen (und logischen) Vermittlung tendenziell verdrängt (vgl. Demirović 2004). Der politische Anspruch auf eine sozial-ontologische Trennbarkeit zwischen Emanzipation und Unterdrückung misslingt aber gerade dort, wo auf ihm in der postoperaistischen Theorie beharrt wird. Was dem politisch-widerständigen Konzept der immateriellen Arbeit ermangelt, ist eine normativ begründete Distinktion, welche es vermag, die im politischen Sinn emanzipatorischen Aspekte der immateriellen Arbeit von ihrem kapitalistischen Zerrbild klar zu unterscheiden. Immaterielle Arbeit als solche lässt sich nicht als objektiver Verwirklichungsgarant eines auf Befreiung ausgerichteten politischen Geschichtsverständnisses fassen. Immaterielle Arbeit als ein per se emanzipatorisches gesellschaftliches Verhältnis zu verstehen, gelingt nur im subjektiven Bereich einer zuschreibenden Auslegungspraxis. Dies betrifft hier vor allem eine Auslegungspraxis, die einseitig bleiben muss, da sie die dem Konzept der immateriellen Arbeit inhärenten Momente von Herrschaft, Gewalt und Unterdrückung ihrerseits unterdrücken oder verdrängen muss. Als ein wesentlich subjektives theoretisches Konzept fehlt der immateriellen Arbeit die nötige historische Durchsetzungskraft, derer es bedürfte, um sie als einen objektiven geschichtlichen Zug der Befreiung im Rahmen einer politischen Theorie entwerfen zu können. Zu seinem eigenen Leidwesen kippt daher das politische Unternehmen Negris und Hardts an seinen theoretisch fragwürdigsten Stellen um in eine Predigt, oder ärger, in einen Werbetext für das Gemeinsam-Werden des lebendigen Fleisches der Multitude.

\section{Literatur}

Atzert, Thomas/Müller, Jost (Hg.) (2004): Immaterielle Arbeit und imperiale Souveränität. Analysen und Diskussionen zu Empire, Münster: Verlag Westfälisches Dampfboot.

Boltanski, Luc/Chiapello, Ève (2003): Der neue Geist des Kapitalismus, Konstanz: UVK-Verlagsgesellschaft. 
Demirović, Alex (2004): „Vermittlung und Hegemonie“. In: Thomas Atzert/Jost Müller (Hg.), Immaterielle Arbeit und imperiale Souveränität. Analysen und Diskussionen zu Empire, Münster: Verlag Westfälisches Dampfboot, S. 235254.

Hardt, Michael/Negri, Antonio (2003): Empire. Die neue Weltordnung, Frankfurt a.M./New York: Campus.

Hardt, Michael/Negri, Antonio (2004): Multitude. Krieg und Demokratie im Empire, Frankfurt a.M./New York: Campus.

Kels, Peter/Vormbusch Uwe (2005): „Human Resource Management als Feld der Subjektivierung von Arbeit“. In: Arbeitsgruppe SubArO (Hg.), Ökonomie der Subjektivität - Subjektivität der Ökonomie. Forschung aus der HansBöckler-Stiftung, Berlin: Ed. Sigma.

Lazzarato, Maurizio (1998): „Immaterielle Arbeit. Gesellschaftliche Tätigkeit unter den Bedingungen des Postfordismus“. In: Antonio Negri/Maurizio Lazzarato/Paolo Virno (Hg.), Umherschweifende Produzenten. Immaterielle Arbeit und Subversion, Berlin: ID Verlag, 39-52.

Möller, Carola (2005): Immaterielle Arbeit - Die neue Dominante in der Wertschöpfungskette. http://labournet.de/diskussion/arbeit/moeller.html [gefunden am 19.01.06].

Moldaschl, Manfred/Voß, G. Günther (Hg.) (2003)² (2002): Subjektivierung von Arbeit, München: Hampp.

Negri, Antonio (1998): „Autonomie und Separatismus. Netzwerke der Produktion und die Bedeutung des Territoriums im italienischen Nordosten“. In: Antonio Negri/Maurizio Lazzarato/Paolo Virno (Hg.), Umherschweifende Produzenten. Immaterielle Arbeit und Subversion, Berlin: ID Verlag, S. 23-37.

Negri, Antonio/Lazzarato, Maurizio/Virno, Paolo (Hg.) (1998): Umherschweifende Produzenten. Immaterielle Arbeit und Subversion, Berlin: ID Verlag.

Virno, Paul (1998): „Do You Remember Counterrevolution? Soziale Kämpfe und ihr Double“. In: Antonio Negri/Maurizio Lazzarato/Paolo Virno (Hg.), Umherschweifende Produzenten. Immaterielle Arbeit und Subversion, Berlin: ID Verlag, S. 83-111.

Voß, G. Günther/Pongratz, Hans J. (1998): „Der Arbeitskraftunternehmer. Eine neue Grundform der Ware Arbeitskraft?" Kölner Zeitschrift für Soziologie und Sozialpsychologie 50/1, S. 131-158.

Weltz, Alexandra/Pichle, Andreas (Regie) (2004): Antonio Negri - Eine Revolution, die nie endet, Erstausstrahlung im ZDF, von ARTE gesendet am 19.11.2004.

Ziegler, Marc (2004): „Das Empire und der Republikanismus der Menge“. In: Oliver Flügel/Reinhard Heil/Andreas Hetzel (Hg.), Die Rückkehr des Politischen. Demokratietheorien heute, Darmstadt: WBG, S. 293-307. 